PHYSICAL REVIEW D 96, 023518 (2017)

\title{
Gravitational waves in doubly coupled bigravity
}

\author{
Philippe Brax, ${ }^{1, *}$ Anne-Christine Davis, ${ }^{2, \dagger}$ and Johannes Noller ${ }^{3, \ddagger}$ \\ ${ }^{1}$ Institut de Physique Théorique, Université Paris-Saclay, CEA, CNRS, F-91191 Gif/Yvette Cedex, France \\ ${ }^{2}$ DAMTP, Centre for Mathematical Sciences, University of Cambridge, \\ Cambridge CB3 OWA, United Kingdom \\ ${ }^{3}$ Astrophysics, University of Oxford, DWB, Keble Road, Oxford OX1 3RH, United Kingdom
}

(Received 5 April 2017; published 18 July 2017)

\begin{abstract}
We consider gravitational waves from the point of view of both their production and their propagation in doubly coupled bigravity in the metric formalism. In bigravity, the two gravitons are coupled by a nondiagonal mass matrix and show birefrigence. In particular, we find that one of the two gravitons propagates with a speed which differs from one. This deviation is tightly constrained by both the gravitational Cerenkov effect and the energy loss of binary pulsars. When emitted from astrophysical sources, the Jordan frame gravitational wave, which is a linear combination of the two propagating gravitons, has a wave form displaying beats. The best prospect of detecting this phenomenon would come from nano-Hertz interferometric experiments.
\end{abstract}

DOI: 10.1103/PhysRevD.96.023518

\section{INTRODUCTION}

The recent direct detection of gravitational waves $[1,2]$ as predicted by general relativity (GR) [3] one hundred years ago could also serve as a test for alternative theories of gravity. For instance a loose bound on the deviation of the speed of gravitational waves from the speed of light has been extracted from the recent LIGO events [4]. Hence gravitational waves can be used to constrain certain modified gravity theories. Motivated by the late time acceleration of the expansion of the Universe [5,6], models of massive gravity $[7,8]$ have been recently considered where gravity could be the result of the existence of two or more gravitons $[9,10]$. In the case of bigravity, the general case we will consider here is that of doubly coupled models whereby a linear combination of the two gravitons couple to matter $[11,12]$. The gravitational wave phenomenology of the singly coupled case has already been considered $[13,14]$ with the existence of beats in the wave form, which could be detectable by LIGO only if the speed of gravitational waves is extremely close to one. In this paper, we generalize these results to the doubly coupled case, where the amplitude and the phase of the Jordan frame wave is shown to have differing characteristics from the singly coupled case. For instance, the modulation of the GR wave emitted by far away sources does not vanish at large frequency any more.

In bigravity, the two gravitons obey coupled propagation equations with eigenmodes whose speeds deviate from one. In this paper, we focus on the cosmological models where the graviton mass is of order of the Hubble rate now-the

\footnotetext{
*philippe.brax@cea.fr

A.C.Davis@damtp.cam.ac.uk

†noller@physics.ox.ac.uk
}

background and perturbative cosmology of such doubly coupled models has previously been explored in [15-18]. On scales much shorter than the size of the Universe, the mass terms can be neglected and the emission from local sources resembles the one in GR for each individual graviton. We examine the emission from such sources and apply it to the case of binary pulsars. The energy loss is modified compared to GR, which results in a tight bound on the deviation of the speed of gravitational waves at the per mil level [19]. Once emitted and far away from the source, these waves propagate like plane waves which mix and show birefringence, i.e. the Jordan frame gravitational wave can be expressed as an effective propagation wave with a frequency dependent amplitude and phase shift whilst the effective gravitational speed differs from one and is also frequency dependent. The gravitational Cerenkov effect when the effective speed is smaller than the speed of light leads to an even tighter bound [20-22] than the one from binary pulsars.

In view of the recent direct detection of gravitational waves, one may enquire whether gravitational birefringence could be observed. This would require us to disentangle the frequency dependence of the wave form from its amplitude, as the amplitude would be degenerate with the features, such as the masses, of the emitting system. We find that this can only be envisaged at best in the nano-Hertz regime [23] and for small differences between the effective gravitational speed and the speed of light. Otherwise, it is likely that the modulation of the bigravity signal would be averaged out resulting in an undetectable change of the wave amplitude.

The paper is arranged as follows. In Sec. II, we recall the main features of doubly coupled bigravity. In Sec. III, we consider the tensor modes and their emission from local sources. This allows us to use the binary pulsars to put a 
bound on the effective speed of gravity. In Sec. IV, we analyze the propagation from a distant source and in Sec. V the prospect of detecting the effects of gravitational birefringence.

\section{BIGRAVITY}

\section{A. The model}

We consider massive bigravity models coupled to matter in the constrained vielbein formalism, which is equivalent to the metric formulation [24], for energy scales below the strong coupling limit $\Lambda_{3} \sim\left(M_{\mathrm{Pl}} H_{0}^{2}\right)^{1 / 3}$ corresponding to scales larger than $1000 \mathrm{~km}$ 's. ${ }^{1}$ Bigravity can be formulated using two vielbeins $e_{1 \mu}^{a}$ and $e_{2 \mu}^{a}$ [25], which couple to matter with couplings $\beta_{1,2}$ respectively $[11,12]{ }^{2}$ The action comprises three very distinct parts. The first one is simply the Einstein-Hilbert terms for both metrics $g_{\mu \nu}^{1,2}$ built from the two vielbeins

$$
S_{G}=\int d^{4} x e_{1} \frac{R_{1}}{16 \pi G_{N}}+\int d^{4} x e_{2} \frac{R_{2}}{16 \pi G_{N}}
$$

where $R_{1,2}$ are the Ricci scalars built from the respective metrics, and $e_{1,2}$ are the determinants of the vielbeins viewed as $4 \times 4$ matrices. The individual vielbeins $e_{\alpha \mu}^{a}, \alpha=1,2$ are constrained to satisfy the symmetric condition

$$
e_{1 \mu}^{a} e_{2 \nu}^{b} \eta_{a b}=e_{1 \nu}^{a} e_{2 \mu}^{b} \eta_{a b}
$$

which we explicitly enforce. This ensures the equivalence with doubly coupled bigravity in the metric formulation, in particular all the terms in the action can be written in terms of the two individual metrics $g_{\mu \nu}^{\alpha}, \alpha=1,2$ defined by

$$
g_{\mu \nu}^{\alpha}=\eta_{a b} e_{\alpha \mu}^{a} e_{\alpha \nu}^{b} .
$$

Matter, i.e. all the fields of the standard model of particle physics, couple to the Jordan metric

$$
g_{\mu \nu}=\eta_{a b} e_{\mu}^{a} e_{\nu}^{b}
$$

built from the local frame [12]

\footnotetext{
${ }^{1}$ Technically speaking this is the scale where perturbative unitarity is lost for fluctuations around Minkowski. While this is therefore an excellent guess for the cutoff scale, whether full unitarity is lost at $\Lambda_{3}$, i.e. whether this scale is a strict cutoff, is still not known. Also note that, for backgrounds different to Minkowski, this scale will get redressed. For example ratios of the scale factors in the theory will modify this scale, when FRW backgrounds are chosen for both metrics.

${ }^{2}$ Note that in general other consistent nonderivative matter couplings exist [26], but when enforcing the symmetric vielbein condition (as we do here) the couplings of $[11,12]$ are the unique consistent matter couplings [26-30]. In this context also note the derivative couplings of [31].
}

$$
e_{\mu}^{a}=\beta_{1} e_{1 \mu}^{a}+\beta_{2} e_{2 \mu}^{a}
$$

where $a$ is a local Lorentz index and $\mu$ the global coordinate index associated with the one forms $e^{a}=e_{\mu}^{a} d x^{\mu}$. The Jordan metric $g_{\mu \nu}$ is explicitly related to the $g_{\mu \nu}^{\alpha}$ 's by

$$
g_{\mu \nu}=\beta_{1}^{2} g_{\mu \nu}^{1}+\beta_{1} \beta_{2} Y_{\mu \nu}+\beta_{2}^{2} g_{\mu \nu}^{2}
$$

where we have defined the symmetric tensor

$$
Y_{\mu \nu}=\eta_{a b}\left(e_{1 \mu}^{a} e_{2 \nu}^{b}+e_{2 \mu}^{a} e_{1 \nu}^{b}\right)
$$

which is also directly linked to $g_{\mu \nu}^{\alpha}, \alpha=1,2$ as the symmetric condition is enforced.

Matter fields $\psi_{i}$ are (minimally) coupled to $g_{\mu \nu}$ and the matter action involves the coupling of the matter fields $\psi_{i}$ 's to the Jordan metric $g_{\mu \nu}$

$$
S_{m}\left(\psi_{i}, g_{\mu \nu}\right) .
$$

Massive bigravity involves also a potential term $[9,10,25]$

$$
S_{V}=\Lambda^{4} \sum_{i j k l} m^{i j k l} \int d^{4} x \epsilon_{a b c d} \epsilon^{\mu \nu \rho \sigma} e_{i \mu}^{a} e_{j \nu}^{b} e_{k \rho}^{c} e_{l \sigma}^{d}
$$

where

$$
\Lambda^{4}=m^{2} M_{\mathrm{Pl}}^{2}
$$

and $m$ is related to the graviton mass while the dimensionless and fully symmetric tensor $m^{i j k l}$ involves five real coupling constants of order one. Both the matter coupling and the potential terms can be expressed as a function of the individual metrics $g_{\mu \nu}^{\alpha}$.

The Jordan frame energy-momentum tensor is defined by

$$
T_{\mu \nu}=-\frac{2}{e} \frac{\delta S_{m}}{\delta g^{\mu \nu}},
$$

which is obtained by varying the matter action with respect to the Jordan metric, i.e. not with respect to the two metrics $g_{\mu \nu}^{\alpha}$. The Einstein equations for both metrics which follow from this setting read

$$
G_{\mu \nu}^{1}=8 \pi G_{N}\left(T_{\mu \nu}^{1}+\mathcal{T}_{\mu \nu}^{1}\right)
$$

and

$$
G_{\mu \nu}^{2}=8 \pi G_{N}\left(T_{\mu \nu}^{2}+\mathcal{T}_{\mu \nu}^{2}\right)
$$

where we have introduced the tensors

$$
T_{\mu \nu}^{\alpha}=-\frac{2}{e_{\alpha}} \frac{\delta S_{m}}{\delta g_{\alpha}^{\mu \nu}}, \quad \mathcal{T}_{\mu \nu}^{\alpha}=-\frac{2}{e_{\alpha}} \frac{\delta S_{V}}{\delta g_{\alpha}^{\mu \nu}}
$$

from which both the background cosmology and the gravitational wave equations can be deduced. In the following, we 
will recall how the background cosmological solutions appear. For gravitational waves, we will derive them by directly using the Lagrangian of bigravity at the second order level in the gravitational perturbations.

\section{B. Cosmological background}

The previous model can be specialized by choosing the cosmological ansatz for the metrics

$$
d s_{1}^{2}=a_{1}^{2}\left(-d \eta^{2}+d x^{2}\right)
$$

and

$$
d s_{2}^{2}=a_{2}^{2}\left(-b^{2} d \eta^{2}+d x^{2}\right)
$$

where the ratio between the lapse functions $b^{2}$ plays a crucial role in the modification of gravity induced by the bigravity models. We consider the coupling of bigravity to a perfect fluid defined by the energy-momentum tensor

$$
T^{\mu \nu}=(\rho+p) u^{\mu} u^{\nu}+p g^{\mu \nu}
$$

where the 4-vector $u^{\mu}$ is $u^{\mu}=\frac{d x^{\mu}}{d \tau_{J}}$ and the proper time in the Jordan frame is simply $d \tau_{J}^{2}=-g_{\mu \nu} d x^{\mu} d x^{\nu}$. Using the fact that the Jordan interval is given by

$d s^{2}=-\left(\beta_{1} a_{1}+\beta_{2} b a_{2}\right)^{2} d \eta^{2}+\left(\beta_{1} a_{1}+\beta_{2} a_{2}\right)^{2} d x^{2}$

we can identify the Jordan frame scale factor

$$
a_{J}=\beta_{1} a_{1}+\beta_{2} a_{2}
$$

and the conformal times

$$
d \eta_{1}=d \eta, \quad d \eta_{2}=b d \eta
$$

when the Jordan conformal time is

$$
d \eta_{J}=\frac{\beta_{1} a_{1}+\beta_{2} b a_{2}}{\beta_{1} a_{1}+\beta_{2} a_{2}} d \eta .
$$

Matter is conserved in the Jordan frame, as follows from the residual diffeomorphism invariance of the matter action, implying that

$$
\frac{d \rho}{d \eta_{J}}+3 a_{J} H_{J}(\rho+p)=0
$$

where the Jordan frame Hubble rate is identified with

$H_{J} \equiv \frac{d a_{J}}{a_{J}^{2} d \eta_{J}}=\frac{1}{\left(\beta_{1} a_{1}+\beta_{2} b a_{2}\right) a_{J}}\left(\beta_{1} a_{1}^{2} H_{1}+\beta_{2} a_{2}^{2} H_{2}\right)$

and we have introduced the two Hubble rates $H_{1}=$ $\frac{d a_{1}}{a_{1}^{2} d \eta_{1}} \equiv \frac{d a_{1}}{a_{1}^{2} d \eta}, H_{2}=\frac{d a_{2}}{a_{2}^{2} d \eta}$. The cosmological dynamics are governed by the two Friedmann equations

$$
3 H_{1}^{2} M_{\mathrm{Pl}}^{2}=\beta_{1} \frac{a_{J}^{3}}{a_{1}^{3}} \rho+24 \Lambda^{4} m^{1 j k l} \frac{a_{j} a_{k} a_{l}}{a_{1}^{3}},
$$

and

$$
\frac{3 H_{2}^{2} M_{\mathrm{Pl}}^{2}}{b^{2}}=\beta_{2} \frac{a_{J}^{3}}{a_{2}^{3}} \rho+24 \Lambda^{4} m^{2 j k l} \frac{a_{j} a_{k} a_{l}}{a_{2}^{3}} .
$$

These equations have two types of solutions. Here we consider only the branch of solutions which satisfies the constraint

$$
b=\frac{a_{2} H_{2}}{a_{1} H_{1}} .
$$

This choice is dictated by the fact that the standard background Friedmann-Robertson-Walker (FRW) cosmology in the radiation and matter eras can be retrieved along this branch. The other branch suffers from inconsistencies at the background level in the radiation era [16]. It turns out that the dynamics simplify both at late and early times. When dark energy is negligible, i.e. in the radiation and matter eras, we have that the ratio $X=\frac{a_{2}}{a_{1}}$ converges to a constant

$$
X \rightarrow X_{m}=\frac{\beta_{2}}{\beta_{1}}
$$

and in the asymptotic future when dark energy dominates, i.e. when the terms in $\Lambda^{4}$ in both Friedmann equations (2.24) and (2.25) are dominant, we have that

$$
X \rightarrow X_{d}
$$

where

$$
X_{d}=\frac{m^{2 j k l} a_{j} a_{k} a_{l}}{m^{1 j k l} a_{j} a_{k} a_{l}} .
$$

In both cases we have that

$$
b=1 .
$$

${ }^{3}$ Between these eras, and in particular now, $b \neq 1$ and $X$ is not equal to its asymptotic value [24], see Fig. 1. Although we have not performed a thorough investigation of the

\footnotetext{
${ }^{3}$ It is interesting to notice that although $X_{m}$ diverges formally when $\beta_{1} \rightarrow 0$, the physical Hubble rate in the Jordan frame $H_{J}$ in both the matter and radiation eras is given by

$$
H_{J}^{2}=\frac{\beta_{1}^{2}+\beta_{2}^{2}}{3 m_{\mathrm{Pl}}^{2}} \rho
$$

which is well-behaved and shows that the effective Newton constant is rescaled by a factor $\beta_{1}^{2}+\beta_{2}^{2}$.
} 


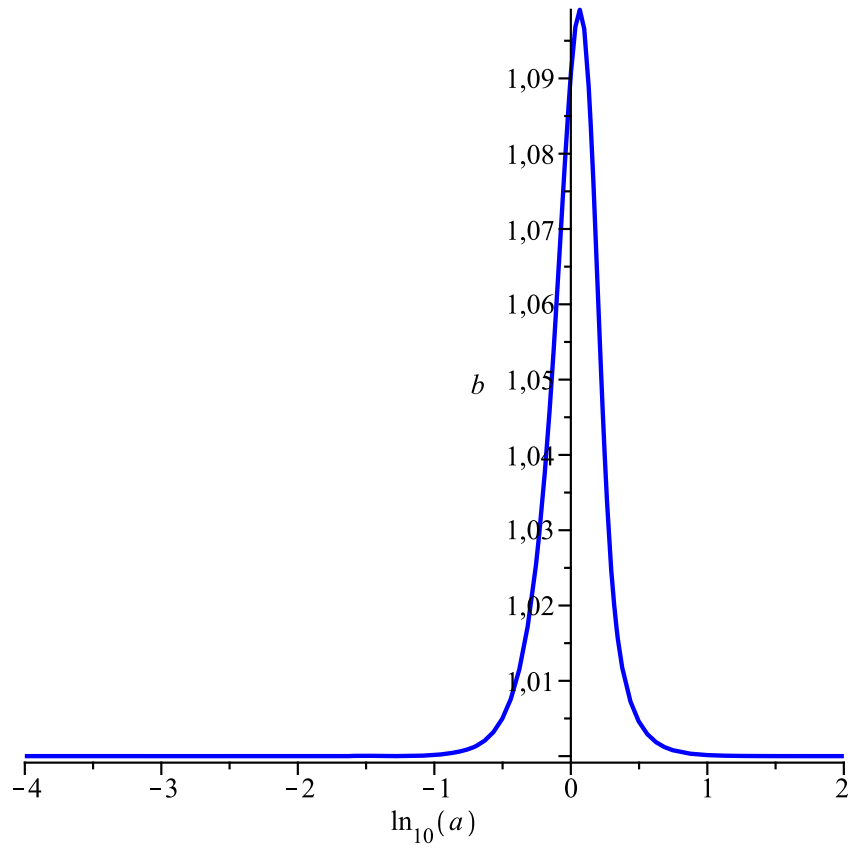

FIG. 1. The variation of $b$ as a function of $\ln a_{J}$ for a model where all the $m_{i j k l}=1, \beta_{1}=1.1$ and $\beta_{2}=1$. In the recent past of the Universe, $b$ starts deviating from 1 before settling back to one in the far future.

dependence of the maximal deviation of $b$ from unity as a function of the parameter space of the model, yet some conclusions can be drawn easily. For instance, $b$ deviates from unity only when the two couplings $\beta_{1,2}$ differ or the five constants $m_{i j k l}$ differ too. In [24], some examples where the couplings or the constants $m_{i j k l}$ differ were considered. What we find is that the percentage of deviation of $b$ from unity is roughly given by the percentage of difference of the couplings or the constants $m_{i j k l}$ respectively. In the numerical example of Fig. 1, the two couplings deviate by $10 \%$ which is also the maximal deviation of $b$ from unity. This deviation will prove to be particularly important for gravitational waves as the effective speed of propagation deviates from one when $b \neq 1$, i.e. we can expect to have non-standard gravitational wave propagation in the recent Universe.

\section{TENSOR MODES: EMISSION AND PROPAGATION}

\section{A. Propagation equations in vacuum}

There are two gravitons in bigravity models. They can be characterized using the tensor perturbations of the two vielbeins

$$
\delta e_{j}^{\alpha i}=a_{\alpha} h_{\alpha j}^{i}
$$

where $\alpha=1,2$ and $h_{\alpha j}^{i}$ is a symmetric transverse and traceless tensor with two degrees of freedom. In the rest of this paper, we do not consider scalar and vector perturbations and only concentrate on the helicity two parts of the perturbations [24]. The potential term of bigravity induces a mass term for the gravitons which reads

$$
M_{\alpha \beta}^{2}\left(a_{\gamma}\right)=-24 m^{2}\left(b_{\alpha} b_{\beta}\right)^{1 / 2} m_{\alpha \beta}\left(a_{\gamma}\right)
$$

which is a symmetric matrix of order $m^{2}$ where

$$
m_{\alpha \beta}\left(a_{\gamma}\right)=\sum_{\gamma \delta} m_{\alpha \beta \gamma \delta} \tilde{a}_{\gamma} a_{\delta}
$$

and $\tilde{a}_{\alpha}=b_{\alpha} a_{\alpha}$ with $b_{1}=1$ and $b_{2}=b$. This mass matrix is solely determined by the overall scale $m$ and the constants $m_{\alpha \beta \gamma \delta}$ from the potential term of bigravity. Here we assume that these constants are all naturally chosen to be of order one implying that the whole mass matrix is of order $m$, i.e. of the order of the Hubble rate now $H_{0}$. We have normalized the tensor modes according to

$$
\bar{h}_{i j}^{1}=M_{\mathrm{Pl}} a_{1} h_{i j}^{1}, \quad \bar{h}_{i j}^{2}=M_{\mathrm{Pl}} \frac{a_{2}}{b^{1 / 2}} h_{i j}^{2} .
$$

Notice that the mass matrix is not diagonal and evolves with time. This induces a mixing of the two gravitons, i.e. birefrigence. The evolution equations for the two gravitons $h_{1}$ and $h_{2}$ in vacuum can be deduced from the action expanded to second order in the perturbations and read

$\frac{d^{2} \bar{h}_{1}}{d \eta^{2}}-\Delta \bar{h}_{1}+\left(M_{11}^{2}\left(a_{\gamma}\right)-\frac{1}{a_{1}} \frac{d^{2} a_{1}}{d \eta^{2}}\right) \bar{h}_{1}+M_{12}^{2}\left(a_{\gamma}\right) \bar{h}_{2}=0$

and

$$
\begin{aligned}
& \frac{d^{2} \bar{h}_{2}}{d \eta^{2}}-b^{2} \Delta \bar{h}_{2}+\left(M_{22}^{2}\left(a_{\gamma}\right)-\frac{b^{1 / 2}}{a_{2}} \frac{d^{2}\left(a_{2} b^{-1 / 2}\right)}{d \eta^{2}}\right) \overline{h_{2}} \\
& \quad+M_{21}^{2}\left(a_{\gamma}\right) \bar{h}_{1}=0 .
\end{aligned}
$$

The coupling between the two gravitons will induce beats in the Jordan gravitational waves. This follows from the fact that matter couples to the Jordan frame combination of gravitons

$$
a_{J} h_{j J}^{i}=\beta_{1} a_{1} h_{j 1}^{i}+\beta_{2} a_{2} h_{j 2}^{i}
$$

and one can see that this evolves with time, i.e. matter couples to different gravitons in the history of the Universe. Let us now consider the propagation when matter sources are taken into account.

\section{B. Gravitational waves from local sources}

Let us now consider a gravitational source and the way gravitational waves are emitted. This can be conveniently 
analysed starting from the action of the two gravitons coupled to matter. Let us recall first how this operates in general relativity. The action involves

$$
\begin{aligned}
\mathcal{L}_{\mathrm{GR}}= & \frac{1}{2}\left(\frac{d \bar{h}_{i j}}{d \eta} \frac{d \bar{h}^{i j}}{d \eta}-\vec{\nabla} \bar{h}_{i j} \vec{\nabla} \bar{h}^{i j}+\frac{1}{a} \frac{d^{2} a}{d \eta^{2}} \bar{h}^{i j} \bar{h}_{i j}\right) \\
& +\frac{a}{M_{\mathrm{Pl}}} \bar{h}^{i j} \bar{T}_{i j}
\end{aligned}
$$

where $\bar{T}_{i j}=T_{i j}-\frac{\delta_{i j}}{3} T$ and indices are raised with $\delta^{i j}$. The gravitational equation becomes

$$
\frac{d^{2} \bar{h}_{i j}}{d \eta^{2}}-\Delta \bar{h}_{i j}-\frac{1}{a} \frac{d^{2} a}{d \eta^{2}} \bar{h}_{i j}=\frac{a}{M_{\mathrm{Pl}}} \bar{T}_{i j}
$$

where here $a$ is the scale factor of the FRW Universe and $\bar{T}_{i j}$ the traceless part of the spatial energy momentum tensor. Notice that in general relativity we have $8 \pi G_{N}=$ $M_{\mathrm{Pl}}^{-2}$. In bigravity, matter couples to the Jordan frame energy-momentum tensor too via

$$
S_{\mathrm{in}}=\int d^{4} x \tilde{a}_{J}\left(\beta_{1} \bar{h}_{1}^{i j}+\beta_{2} b^{1 / 2} \bar{h}_{2}^{i j}\right) \bar{T}_{i j} .
$$

As a result the coupled gravitational equations become

$$
\begin{aligned}
& \frac{d^{2} \bar{h}_{i j}^{1}}{d \eta^{2}}-\Delta \bar{h}_{i j}^{1}+\left(M_{11}^{2}\left(a_{\gamma}\right)-\frac{1}{a_{1}} \frac{d^{2} a_{1}}{d \eta^{2}}\right) \bar{h}_{i j}^{1} \\
& \quad+M_{12}^{2}\left(a_{\gamma}\right) \bar{h}_{i j}^{2}=\beta_{1} \frac{\tilde{a}_{J}}{M_{\mathrm{Pl}}} \bar{T}_{i j}
\end{aligned}
$$

and

$$
\begin{aligned}
& \frac{d^{2} \bar{h}_{i j}^{2}}{d \eta^{2}}-b^{2} \Delta \bar{h}_{i j}^{2}+\left(M_{22}^{2}\left(a_{\gamma}\right)-\frac{b^{1 / 2}}{a_{2}} \frac{d^{2}\left(a_{2} b^{-1 / 2}\right)}{d \eta^{2}}\right) \bar{h}_{i j}^{2} \\
& \quad+M_{21}^{2}\left(a_{\gamma}\right) \bar{h}_{i j}^{1}=\beta_{2} \frac{b^{1 / 2} \tilde{a}_{J}}{M_{\mathrm{Pl}}} \bar{T}_{i j} .
\end{aligned}
$$

Notice that the source terms involve both couplings to matter $\beta_{1,2}$ and complement the propagation equations in vacuum (3.11) and (3.12). In the following, we shall be only interested in waves which propagate on distances for which one can neglect the effects of the cosmological evolution. The generalization to the cosmological case can be easily analyzed too and is left for future work. We will also assume that the waves are emitted at a redshift corresponding to $a_{J}$ in bigravity and $a_{\mathrm{GR}}$ in $\Lambda$-CDM. Both in bigravity and in GR, the scale factors $a_{J}$ and $a_{\mathrm{GR}}$ are normalized to be one now. As a result, the metrics read

$$
d s_{\mathrm{GR}}^{2} \sim-d t_{\mathrm{GR}}^{2}+d \vec{r}_{\mathrm{GR}}^{2}
$$

and

$$
d s_{J}^{2} \sim-d t_{J}^{2}+d \vec{r}_{J}^{2}
$$

where $d t_{J}=\tilde{a}_{J} d \eta$ and $d t_{\mathrm{GR}}=a_{\mathrm{GR}} d \eta$. Moreover we have $d \vec{r}_{J}=a_{J} d \vec{x}$ and $d \vec{r}_{\mathrm{GR}}=a_{\mathrm{GR}} d \vec{x}$. As $a_{\mathrm{GR}} \sim a_{J} \sim 1$ in the recent past of the Universe, the only difference between the two metrics now comes from the different clocks with $a_{J}=\beta_{1} a_{1}+\beta_{2} a_{2}$ and $\tilde{a}_{J}=\beta_{1} a_{1}+\beta_{2} b a_{2}$ when $b \neq 1$. We also assume that the waves can be well approximated by plane waves sufficiently far from the source.

\section{Emission from binary pulsars}

The emission of gravitational waves by binary pulsars leads to tight constraints on modified gravity. Here the emission takes places on scales much smaller than the inverse mass of the gravitons, i.e. less than the size of the Universe. The perturbative equations that we adopt are only valid at low energy corresponding to time scales larger than the inverse cut-off $\Lambda_{3}^{-1} \sim 10^{-2} \mathrm{~s}$. As the typical period of binary pulsars is of the order of a few hours, the description which follows, where the emission of gravitational waves is considered in bigravity, can be applied to binary pulsars. The wave equations in the emission region therefore simplify

$$
\frac{d^{2} \bar{h}_{i j}^{1}}{d \eta^{2}}-\Delta \bar{h}_{i j}^{1}=\beta_{1} \frac{\tilde{a}_{J}}{M_{\mathrm{Pl}}} \bar{T}_{i j}
$$

and

$$
\frac{d^{2} \bar{h}_{i j}^{2}}{d \eta^{2}}-b^{2} \Delta \bar{h}_{i j}^{2}=\beta_{2} \frac{\tilde{a}_{J} b^{1 / 2}}{M_{\mathrm{Pl}}} \bar{T}_{i j}
$$

The Newtonian trajectories of the binary objects are not modified in doubly-coupled bigravity (see Sec. Vof [24]) and here we consider that this is still a reasonable assumption in the case of compact objects with Newtonian potentials $\Phi_{N} \lesssim 0.1$. In the quasistatic limit and in a Minkowski background this follows from the fact that the extra scalar field in the spectrum of bigravity decouples from matter in the doubly coupled case. This can also be seen in the decoupling limit where the coupling of the scalar field $\pi$ appears in the form $\partial_{\mu} \partial_{\nu} \pi T^{\mu \nu}$ which does not lead to a source term for $\pi$ and therefore implies that there is no new force acting on massive objects. The solutions to the wave equations are simply

$\bar{h}_{i j}^{1}(\vec{x}, \eta)=\beta_{1} \frac{\tilde{a}_{J}}{4 \pi M_{\mathrm{Pl}}} \Lambda_{i j}^{k l} \int d^{3} y \frac{\bar{T}_{k l}(\eta-|x-y|, y)}{|x-y|}$

in conformal coordinates and

$\bar{h}_{i j}^{2}(\vec{x}, \eta)=\beta_{2} \frac{\tilde{a}_{J} b^{1 / 2}}{4 \pi M_{\mathrm{Pl}}} \Lambda_{i j}^{k l} \int d^{3} y \frac{\bar{T}_{i j}\left(\eta-\frac{|x-y|}{b}, y\right)}{|x-y|}$.

Assuming that the energy-momentum tensor of the source has compact support and $|x| \gg|y|$ we have the approximation 
$\bar{h}_{i j}^{1}(\vec{x}, \eta)=\beta_{1} \frac{\tilde{a}_{J}}{4 \pi M_{\mathrm{Pl}}} \frac{1}{|x|} \Lambda_{i j}^{k l} \int d^{3} y \bar{T}_{i j}(\eta-|x|, y)$

in conformal coordinates and

$\bar{h}_{i j}^{2}(\vec{x}, \eta)=\beta_{2} \frac{\tilde{a}_{J} b^{1 / 2}}{4 \pi M_{\mathrm{Pl}}} \frac{1}{|x|} \Lambda_{i j}^{k l} \int d^{3} y \bar{T}_{k l}\left(\eta-\frac{|x|}{b}, y\right)$.

Using the identity

$\frac{d^{2}}{d \eta^{2}} \int d^{3} y y^{i} y^{j} T^{00}(\eta-|x|, y)=2 \int d^{3} y T^{i j}(\eta-|x|, y)$

for the conserved energy-momentum in the Jordan frame, we find that

$$
\bar{h}_{\alpha}^{i j}(\vec{x}, \eta)=\beta_{\alpha} b_{\alpha}^{1 / 2} \frac{a_{J}^{4}}{\tilde{a}_{J} 8 \pi M_{\mathrm{Pl}}} \frac{1}{|x|} \Lambda_{k l}^{i j} \frac{d^{2} \bar{I}^{k l}}{d \eta^{2}}
$$

where indices are raised and lowered with the flat $\delta^{i j}$. This implies that

$$
\bar{h}_{\alpha}^{i j}(\vec{x}, \eta)=\frac{\beta_{\alpha} b_{\alpha}^{1 / 2}}{\beta_{1}^{2}+\beta_{2}^{2}} \frac{a_{J}^{4}}{\tilde{a}_{J} a_{\mathrm{GR}}^{3}} \bar{h}_{\mathrm{GR}}^{i j}(\vec{x}, \eta) .
$$

where we have used the fact that the local and cosmological Newton constants in bigravity models is [24]

$$
G_{\text {local }}=G_{\text {cosmo }}=\left(\beta_{1}^{2}+\beta_{2}^{2}\right) G_{N}
$$

where $G_{N}$ is only a parameter in the action. We have introduced the usual tensor

$$
\Lambda_{i j}^{k l}=P_{i}^{k} P_{l}^{j}-\frac{1}{2} P_{i j} P^{k l}
$$

where

$$
P_{i j}=\delta_{i j}-n_{i} n_{j}
$$

which is the projector orthogonal to the propagation vector $n_{i}$. The tensor $\Lambda_{i j}^{k l}$ enforces the transverse traceless condition. We also have the Jordan combination

$$
h_{i j}^{J}(\vec{x}, \eta)=G_{\text {cosmo }} \frac{a_{J}^{3}}{\tilde{a}_{J}} \frac{\beta_{1}^{2}+b \beta_{2}^{2}}{\beta_{1}^{2}+\beta_{2}^{2}} \frac{1}{|x|} \Lambda_{i j k l} \frac{d^{2} \bar{I}^{k l}}{d \eta^{2}}
$$

where, in terms of the matter density $\rho$,

$$
\bar{I}^{i j}=\int d^{3} y\left(y^{i} y^{j}-\frac{1}{3} \delta^{i j}|y|^{2}\right) \rho(\eta-|x|, y)
$$

to leading order in a multipolar expansion. We have assumed that $b$ is very close to unity.
The energy flux emitted by the object can be evaluated as in [32] where it is the energy given to matter minus the one that matter radiates subsequently. As the gravitational waves couple to matter in the Jordan frame, this depends only on the derivatives of $h_{J}$

$$
F=\frac{1}{8 \pi a_{J}^{4}}\left\langle\left(\frac{d h_{i j}}{d t_{J}}\right)^{2}\right\rangle
$$

where the average is a time average. The energy loss is given

$$
\frac{d E}{d t_{J}}=-\int F a_{J}^{2}|x|^{2} d \Omega
$$

and therefore

$$
\frac{d E}{d t_{J}}=-\frac{a_{J}^{4}}{2 \tilde{a}_{J}^{4}} G_{\text {cosmo }}^{2}\left(\frac{\beta_{1}^{2}+b \beta_{2}^{2}}{\beta_{1}^{2}+\beta_{2}^{2}}\right)^{2}\left\langle\left(\dddot{\mathcal{I}}_{i j}\right)^{2}\right\rangle
$$

where the time derivatives are with respect to $\eta$. As a result

$$
\frac{d E}{d t_{J}}=\left(\frac{\beta_{1}^{2}+b \beta_{2}^{2}}{\beta_{1}^{2}+\beta_{2}^{2}}\right)^{2} \frac{a_{J}^{4}}{\tilde{a}_{J}^{4}} \frac{d E}{d t_{\mathrm{GR}}} .
$$

Notice that the deviation from the GR result is only present when $b \neq 1$. As we have already recalled, this is the case in the present Universe. There is a tight constraint on the possible difference with GR and it reads [19]

$$
0.995<\left(\frac{\beta_{1}^{2}+b \beta_{2}^{2}}{\beta_{1}^{2}+\beta_{2}^{2}}\right)^{2} \frac{a_{J}^{4}}{\tilde{a}_{J}^{4}}<1
$$

which gives a constraint on $b$ at the $10^{-3}$ level. In the following, we shall investigate what happens to the propagation of the gravitational waves when $b$ is constrained at a level tighter than one per mil.

Our calculation has taken into account the quadrupolar emission from binary pulsars. In this case, the distance between the two stars is much larger than the cutoff distance of bigravity and our calculation is valid where the two stars are considered to be orbiting subject to Newton's law.

On the other hand, since the stars themselves (typically neutron stars) are much smaller than the cutoff scale of bigravity, their dynamics will most likely be sensitive to details of the UV completion of the theory. For example, the additional decoupled scalar degree of freedom of doubly coupled bigravity [24], which naively becomes a ghost below the cutoff distance, may correspond to a healthy degree of freedom in the UV-completed theory and lead to stars acquiring scalar charges. This would lead to the possible emission of dipolar gravitational waves $[33,34]$. Another phenomenon which is beyond the present treatment corresponds to the last phase of the merger between two black holes when their distance falls below 
$1000 \mathrm{~km}$ 's. The calculation of the emission spectrum cannot be tackled using the models described here. All these effects are beyond the present work.

\section{PROPAGATION}

Let us come back to the propagation of gravitational waves in empty space, when the initial wave is due to a localized source which is far-away and the waves can be considered to be plane-waves.

\section{A. Eigenmodes}

It is convenient to define the effective mass matrix

$$
\tilde{M}^{2}=\left(\begin{array}{cc}
M_{11}^{2}-\frac{1}{a_{1}} \frac{d^{2} a_{1}}{d \eta^{2}} & M_{12}^{2} \\
M_{12}^{2} & \left.M_{22}^{2}-\frac{b^{1 / 2}}{a_{2}} \frac{d^{2}\left(a_{2} b^{-1 / 2}\right)}{d \eta^{2}}\right)
\end{array}\right) .
$$

The two propagation equations for gravitons have two eigenmodes which can be described by

$$
h_{\alpha \pm}=A_{\alpha \pm} e^{i\left(\omega_{ \pm} t-i \vec{k} \cdot \vec{x}\right)}
$$

where $\alpha=1,2$. The eigenfrequencies are given by the quartic dispersion relation

$$
\begin{aligned}
& \omega^{4}-\omega^{2}\left(\left(1+b^{2}\right) \vec{k}^{2}+\tilde{M}_{11}^{2}+\tilde{M}_{22}^{2}\right)-\tilde{M}_{12}^{2} \tilde{M}_{21}^{2} \\
& \quad+\left(\vec{k}^{2}+\tilde{M}_{11}^{2}\right)\left(\beta^{2} \vec{k}^{2}+\tilde{M}_{22}^{2}\right)=0 .
\end{aligned}
$$

Defining the discriminant

$$
\Delta=\left(\left(1-b^{2}\right) \vec{k}^{2}+\tilde{M}_{11}^{2}-\tilde{M}_{22}^{2}\right)^{2}+4 \tilde{M}_{12}^{2} \tilde{M}_{21}^{2}
$$

we have the two eigenfrequencies

$$
\omega_{ \pm}^{2}=\frac{\omega^{2}\left(\vec{k}^{2}+\tilde{M}_{11}^{2}+b^{2} \vec{k}^{2}+\tilde{M}_{22}^{2}\right) \pm \sqrt{\Delta}}{2} .
$$

We only consider gravitational waves such that $\vec{k}^{2} \gg \tilde{M}_{i j}^{2}$ as the mass matrix elements are of order $H_{0}$ and astrophysical waves are much more energetic than this. As a result we obtain the expansion

$$
\begin{gathered}
\omega_{+}^{2} \sim \vec{k}^{2}+\tilde{M}_{11}^{2}+\frac{\tilde{M}_{12}^{4}}{\left(1-b^{2}\right) \vec{k}^{2}+\tilde{M}_{11}^{2}-\tilde{M}_{22}^{2}} \\
\omega_{-}^{2} \sim b^{2} \vec{k}^{2}+\tilde{M}_{22}^{2}-\frac{\tilde{M}_{12}^{4}}{\left(1-b^{2}\right) \vec{k}^{2}+\tilde{M}_{11}^{2}-\tilde{M}_{22}^{2}} .
\end{gathered}
$$

The two eigenmodes are then obtained as

$$
h_{-}=h_{2}-C h_{1}, \quad h_{+}=h_{1}+C h_{2}
$$

in terms of $h_{1,2}$ where

$$
C=\frac{\tilde{M}_{12}^{2}}{\left(1-b^{2}\right) k^{2}+\tilde{M}_{11}^{2}-\tilde{M}_{22}^{2}} .
$$

Equivalently we have

$$
h_{1}=\frac{h_{+}-C h_{-}}{1+C^{2}}, \quad h_{2}=\frac{h_{-}+C h_{+}}{1+C^{2}}
$$

which will be useful when defining the Jordan frame graviton. It is convenient to define the characteristic wave number

$$
\bar{k}^{2}=\frac{\left|\tilde{M}_{11}^{2}-\tilde{M}_{22}^{2}\right|}{\left|1-b^{2}\right|} .
$$

Hence when $k \gg \bar{k}, C$ goes to zero in $1 / k^{2}$ whilst when $k \ll \bar{k}, C$ goes to a constant or order one. In fact we have

$$
k \ll \bar{k}, \quad C \sim \frac{\tilde{M}_{12}^{2}}{\tilde{M}_{11}^{2}-\tilde{M}_{22}^{2}}
$$

and

$$
k \gg \bar{k}, \quad C \sim \frac{\tilde{M}_{12}^{2}}{\tilde{M}_{11}^{2}-\tilde{M}_{22}^{2}} \frac{\bar{k}^{2}}{k^{2}} .
$$

The wave number $\bar{k}$ depends on how small the deviation

$$
\frac{\left|\omega_{+}-\omega_{-}\right|}{k} \sim|b-1|
$$

can be, i.e. how small $|b-1|$ is.

The initial conditions for $\bar{h}_{\alpha}$ are related to the waves obtained in general relativity (as the size of the regions where the waves are created is smaller than the cosmological horizon and their energy is very large compared to $H_{0}$ ) scaled by $\left.\frac{\beta_{\alpha} b_{\alpha}^{1 / 2}}{\beta_{1}^{2}+\beta_{2}^{2}} \frac{a_{J}^{4}}{a_{\mathrm{GR}}^{3} \tilde{a}_{J}}\right|_{0}$, see (3.23), i.e.

$$
\bar{h}_{0}^{\alpha}=\left.\frac{\beta_{\alpha} b_{\alpha}^{1 / 2}}{\beta_{1}^{2}+\beta_{2}^{2}} \frac{a_{J}^{4}}{a_{\mathrm{GR}}^{3} \tilde{a}_{J}}\right|_{0} h_{\mathrm{GR}}
$$

where the first denominator comes from the rescaling between the cosmological and local, i.e. physical, Newton constant and the fiducial one in the action. This follows from the calculation in Sec. III of the wave form emitted from a local source. The local source generates the initial wave which then propagate far away in a plane wave approximation. The resulting waves after emission are then simply 


$$
\begin{aligned}
\bar{h}_{1}= & \left(\frac{\beta_{1}}{\beta_{1}^{2}+\beta_{2}^{2}}\left(\frac{e^{i \omega_{+} t}+C^{2} e^{i \omega_{-} t}}{1+C^{2}}\right)\right. \\
& \left.+\frac{\beta_{2} C}{\beta_{1}^{2}+\beta_{2}^{2}}\left(\frac{e^{i \omega_{+} t}-e^{i \omega_{-} t}}{1+C^{2}}\right)\right)\left.e^{-i \vec{k} \cdot \vec{x}} \frac{a_{J}^{4}}{\tilde{a}_{J} a_{\mathrm{GR}}^{3}}\right|_{0} h_{\mathrm{GR}}
\end{aligned}
$$

and

$$
\begin{aligned}
\bar{h}_{2}= & \left(\frac{\beta_{2} b^{1 / 2}}{\beta_{1}^{2}+\beta_{2}^{2}}\left(\frac{e^{i \omega_{-} t}+C^{2} e^{i \omega_{+} t}}{1+C^{2}}\right)\right. \\
& \left.-\frac{\beta_{1} C}{\beta_{1}^{2}+\beta_{2}^{2}}\left(\frac{e^{i \omega_{-} t}-e^{i \omega_{+} t}}{1+C^{2}}\right)\right)\left.e^{-i \vec{k} \cdot \vec{x}} \frac{a_{J}^{4}}{\tilde{a}_{J} a_{\mathrm{GR}}^{3}}\right|_{0} h_{\mathrm{GR}} .
\end{aligned}
$$

As a result we get for the Jordan frame gravitational wave

$$
\bar{h}_{J}=a_{J} h_{J}=\beta_{1} \bar{h}_{1}+\beta_{2} b^{1 / 2} \bar{h}_{2}
$$

the following

$$
\begin{aligned}
\bar{h}_{J}= & \left(\frac{\left(\beta_{1}+\beta_{2} b^{1 / 2} C\right)^{2}}{1+C^{2}} e^{i \omega_{+} t}+\frac{\left(\beta_{2} b^{1 / 2}-\beta_{1} C\right)^{2}}{1+C^{2}} e^{i \omega_{-} t}\right) \\
& \times\left. e^{-i \vec{k} \cdot \vec{x}} \frac{a_{J}^{4}}{\tilde{a}_{J} a_{\mathrm{GR}}^{3}}\right|_{0} \frac{h_{\mathrm{GR}}}{\beta_{1}^{2}+\beta_{2}^{2}}
\end{aligned}
$$

This is the wave-form emitted by a far-away source when the gravitational waves show a birefringent behavior.

\section{B. The effective speed of gravitational waves}

When the $b$ is very close to one, the wave generated by a distant source $A_{J}=\Re\left(h_{J}\right)$ reads

$$
\begin{aligned}
A_{J}= & \left(\frac{\left(\beta_{1}+\beta_{2} C\right)^{2}}{1+C^{2}} \cos \left(i \omega_{+} t-i \vec{k} \cdot \vec{x}\right)\right. \\
& \left.+\frac{\left(\beta_{2}-\beta_{1} C\right)^{2}}{1+C^{2}} \cos \left(i \omega_{-} t-i \vec{k} \cdot \vec{x}\right)\right) \\
& \times \frac{a_{J}^{4}}{\tilde{a}_{J} a_{\mathrm{GR}}^{3}} \frac{h_{\mathrm{GR}}}{\beta_{1}^{2}+\beta_{2}^{2}}
\end{aligned}
$$

where $\omega_{+} \sim \omega_{-}$. Defining $\omega=\frac{\omega_{+}+\omega_{-}}{2}$ and $\Delta \omega=\omega_{-}-\omega_{+}$, we have

$$
\begin{aligned}
A_{J}= & \frac{\left(\beta_{1}+\beta_{2} b^{1 / 2} C\right)^{2}+\left(\beta_{2} b^{1 / 2}-\beta_{1} C\right)^{2}}{1+C^{2}} \\
& \times \cos (\Delta \omega t)[\cos (\omega t-i \vec{k} \cdot \vec{x}) \\
& +\frac{\left(\beta_{1}+\beta_{2} b^{1 / 2} C\right)^{2}-\left(\beta_{2} b^{1 / 2}-\beta_{1} C\right)^{2}}{\left(\beta_{1}+\beta_{2} b^{1 / 2} C\right)^{2}+\left(\beta_{2} b^{1 / 2}-\beta_{1} C\right)^{2}} \\
& \times \tan (\Delta \omega t) \sin (\omega t-i \vec{k} \cdot \vec{x})]\left.\frac{a_{J}^{4}}{\tilde{a}_{J} a_{\mathrm{GR}}^{3}}\right|_{0} \frac{h_{\mathrm{GR}}}{\beta_{1}^{2}+\beta_{2}^{2}} .
\end{aligned}
$$

This represents wave beats compared to the usual wave front of GR. When the two eigenfrequencies satisfy $\Delta \omega t \ll 1$, the wave form can be cast into a propagating wave with a time dependent phase shift

$$
A_{J}=A \cos (\omega t-i \vec{k} \cdot \vec{x}-\delta)
$$

where the amplitude is given by

$$
\begin{aligned}
A= & \frac{\left(\beta_{1}+\beta_{2} b^{1 / 2} C\right)^{2}+\left(\beta_{2} b^{1 / 2}-\beta_{1} C\right)^{2}}{1+C^{2}} \\
& \times\left.\cos (\Delta \omega t) \frac{a_{J}^{4}}{\tilde{a}_{J} a_{\mathrm{GR}}^{3}}\right|_{0} \frac{h_{\mathrm{GR}}}{\beta_{1}^{2}+\beta_{2}^{2}}
\end{aligned}
$$

with a small time dependence and a phase shift

$\delta=\frac{\left(\beta_{1}+\beta_{2} b^{1 / 2} C\right)^{2}-\left(\beta_{2} b^{1 / 2}-\beta_{1} C\right)^{2}}{\left(\beta_{1}+\beta_{2} b^{1 / 2} C\right)^{2}+\left(\beta_{2} b^{1 / 2}-\beta_{1} C\right)^{2}} \tan (\Delta \omega t)$.

The wave propagates with the energy

$$
\omega=c_{T} k+\frac{\tilde{M}_{11}^{2}}{4 k}+\frac{\tilde{M}_{22}^{2}}{4 b k}
$$

in an expansion in $\frac{\tilde{M}_{i j}^{2}}{k^{2}}$ and $k=\sqrt{\vec{k}^{2}}$. We have introduced the effective speed of the gravitational waves

$$
c_{T}=\frac{1+b}{2} .
$$

This effective speed is highly constrained when $b<1$, i.e. when the effective speed is less than the speed of light. Indeed in this case, high energy cosmic rays can emit gravitons in a Cerenkov fashion and this would deplete the number count of cosmic rays on earth. This is not the case if and only if $[20,21]$

$$
(1-b) \lesssim 10^{-17}
$$

One can check that in this case $\Delta \omega d \ll 1$ for sources such that $d \ll 100 \mathrm{Mpc}$. Of course the energy of cosmic rays is higher than the cutoff scale of doubly coupled bigravity so this constraint may be relaxed when considering the UV completion of the model.

As a result, we see that the effective speed of gravitational waves is extremely constrained by observations. In the following section, we will consider the prospects of detecting deviations from GR when the parameter $b$ is so tightly bounded.

\section{The emitted spectrum and detection prospects}

Let us now consider the spectrum of gravitational waves at a distance $d$ from the source. For that, it is convenient to consider the spectrum as obtained from the square of the amplitude 


$$
\left|\bar{h}_{J}\right|^{2}=\left(\left.\frac{a_{J}^{4}}{\tilde{a}_{J} a_{\mathrm{GR}}^{3}}\right|_{0}\right)^{2}\left(\frac{\beta_{1}^{2}+b \beta_{2}^{2}}{\beta_{1}^{2}+\beta_{2}^{2}}\right)^{2}\left(1-4 \frac{\left(\beta_{1}+\beta_{2} b^{1 / 2} C\right)^{2}\left(\beta_{2} b^{1 / 2}-\beta_{1} C\right)^{2}}{\left(\beta_{1}^{2}+b \beta_{2}^{2}\right)^{2}} \sin ^{2}\left(\frac{\left(\omega_{+}-\omega_{-}\right) t}{2}\right)\right)\left|h_{\mathrm{GR}}\right|^{2} .
$$

This means that the signal has a change of amplitude and a time modulation, and that at a time $t=d$

$$
P_{J}(k)=\left(\left.\frac{a_{J}^{4}}{\tilde{a}_{J} a_{\mathrm{GR}}^{3}}\right|_{0} ^{2}\right)^{2}\left(\frac{\beta_{1}^{2}+b \beta_{2}^{2}}{\beta_{1}^{2}+\beta_{2}^{2}}\right)^{2}\left(1-4 \frac{\left(\beta_{1}+\beta_{2} b^{1 / 2} C\right)^{2}\left(\beta_{2} b^{1 / 2}-\beta_{1} C\right)^{2}}{\left(\beta_{1}^{2}+b \beta_{2}^{2}\right)^{2}} \sin ^{2}\left(\frac{\left(\omega_{+}-\omega_{-}\right) d}{2}\right)\right) P_{\mathrm{GR}}(k)
$$

the spectrum is modulated by a frequency dependent prefactor. Let us first connect with the case of singly coupled gravity. When $\beta_{1}$ or $\beta_{2}$ vanishes we find that

$$
\left|\bar{h}_{J}\right|^{2}=\left(\left.\frac{a_{J}^{4}}{\tilde{a}_{J} a_{\mathrm{GR}}^{3}}\right|_{0}\right)^{2}\left(1-4 C^{2} \sin ^{2}\left(\frac{\left(\omega_{+}-\omega_{-}\right) d}{2}\right)\right)\left|h_{\mathrm{GR}}\right|^{2}
$$

where $C$ is constant for $k \lesssim \bar{k}$ and vanishes at large $k$ [14]. This retrieves the known results of the singly coupled case. In the doubly coupled case, the term in $\sin ^{2}$ never vanishes, i.e. this is a clear difference with the singly coupled case.

Let us notice that the modulation should only be effective when the variation of the $\sin ^{2}$ term is not too rapid compared to the frequency of the signal in GR. If this is the case and the averaged $\left\langle\sin ^{2}\right\rangle=1 / 2$ is used, the effects of bigravity are only a change in the amplitude of the signal, i.e. degenerate with the astrophysical features of the emitting system. On the other hand when

$$
|b-1| \lesssim \frac{1}{k_{\exp } d}
$$

where $k_{\exp }$ is the most sensitive frequency of the detecting device, and $d$ the distance to the emitting source, the modulation of the GR signal would be relevant. For sources around $d=100 \mathrm{Mpc}$ and a sensitivity peaking in the nano Hertz regime [23], we can hope to observe effects for $|b-1| \lesssim 10^{-7}$, four orders of magnitude lower than the pulsar bound. The pulsar bound would be probed only by the detection of nano Hertz events in our immediate vicinity around $10 \mathrm{kpc}$.

\section{DISCUSSION AND CONCLUSION}

We have discussed the emission and the propagation of gravitational waves in doubly coupled bigravity. The deviations from GR are essentially governed by one parameter $b$ which differs from one only in the transient cosmological era between the matter era in the past and the future dark energy dominated one. It turns out that the deviation of this parameter from one measures the effective speed of gravitational waves in bigravity. This can be constrained by both the absence of gravitational Cerenkov effect and the energy loss of binary pulsars. As a result, we do not expect that the effective speed of gravitational waves differs from one by more than one per mil. This is still large enough to induce possible modulations of the wave form of the gravitational wave signal in the Jordan frame, i.e. the gravitational wave coupled to matter. The best prospect of detecting this gravitational birefringence would be with nano-Hertz interferometry experiments, and deviations of the gravitational speed up to $10^{-7}$ would be observable from sources further than $100 \mathrm{Mpc}$. Another way of detecting these effects would be to monitor nearby sources of both gravitational and electromagnetic waves and trying to detect a phase difference between these signals [35].

The bound on $|b-1| \lesssim 10^{-3}$ from binary pulsar constraints implies that other effects of bigravity such as a change in the growth of cosmological structures would also be tightly restricted. Indeed, as an order of magnitude, the growth parameters such as $\mu$ and $\Sigma$ deviate from GR as $|b-1|$ and therefore one would not expect effects on structure formation much larger than the percent level. This would have implications for the detection of bigravity effect by future cosmological surveys [36]. The details of this comparison are left for future work.

\section{ACKNOWLEDGMENTS}

We would like to thank Juan Garcia-Bellido and Diego Blas for comments on the manuscript. We are grateful to Nicola Tamanini for remarks and discussions. This project has received funding from the European Union's Horizon 2020 research and innovation programme under the Marie Skłodowska-Curie grant agreement No. 690575, A. C. D. acknowledges partial support from Science and Technology Facilities Council (STFC) under Grants Nos. ST/L000385/1 and ST/L000636/1, J. N. acknowledges support from the Royal Commission for the Exhibition of 1851 and BIPAC. This article is based upon work related to the COST Action CA15117 (CANTATA) supported by COST (European Cooperation in Science and Technology). 
[1] B. P. Abbott et al. (Virgo, LIGO Scientific Collaborations), Observation of Gravitational Waves from a Binary Black Hole Merger, Phys. Rev. Lett. 116, 061102 (2016).

[2] B. P. Abbott et al. (Virgo, LIGO Scientific Collaborations), GW151226: Observation of Gravitational Waves from a 22-Solar-Mass Binary Black Hole Coalescence, Phys. Rev. Lett. 116, 241103 (2016).

[3] A. Buonanno, Gravitational waves, arXiv:0709.4682.

[4] D. Blas, M. M. Ivanov, I. Sawicki, and S. Sibiryakov, On constraining the speed of gravitational waves following GW150914, Pis'ma Zh. Eksp. Teor. Fiz. 103, 708 (2016).

[5] E. J. Copeland, M. Sami, and S. Tsujikawa, Dynamics of dark energy, Int. J. Mod. Phys. D 15, 1753 (2006).

[6] A. Joyce, B. Jain, J. Khoury, and M. Trodden, Beyond the cosmological standard model, Phys. Rep. 568, 1 (2015).

[7] C. de Rham and G. Gabadadze, Generalization of the Fierz-Pauli action, Phys. Rev. D 82, 044020 (2010).

[8] C. de Rham, G. Gabadadze, and A. J. Tolley, Resummation of Massive Gravity, Phys. Rev. Lett. 106, 231101 (2011).

[9] S. Hassan and R. A. Rosen, Resolving the Ghost Problem in Non-Linear Massive Gravity, Phys. Rev. Lett. 108, 041101 (2012).

[10] S. Hassan and R. A. Rosen, Bimetric gravity from ghost-free massive gravity, J. High Energy Phys. 02 (2012) 126.

[11] C. de Rham, L. Heisenberg, and R. H. Ribeiro, On couplings to matter in massive (bi-)gravity, Classical Quantum Gravity 32, 035022 (2015).

[12] J. Noller and S. Melville, The coupling to matter in massive, bi- and multi-gravity, J. Cosmol. Astropart. Phys. 01 (2015) 003.

[13] A. De Felice, T. Nakamura, and T. Tanaka, Possible existence of viable models of bi-gravity with detectable graviton oscillations by gravitational wave detectors, Prog. Theor. Exp. Phys. 2014, 43E01 (2014).

[14] T. Narikawa, K. Ueno, H. Tagoshi, T. Tanaka, N. Kanda, and T. Nakamura, Detectability of bigravity with graviton oscillations using gravitational wave observations, Phys. Rev. D 91, 062007 (2015).

[15] J. Enander, A. R. Solomon, Y. Akrami, and E. Mortsell, Cosmic expansion histories in massive bigravity with symmetric matter coupling, J. Cosmol. Astropart. Phys. 01 (2015) 006.

[16] D. Comelli, M. Crisostomi, K. Koyama, L. Pilo, and G. Tasinato, Cosmology of bigravity with doubly coupled matter, J. Cosmol. Astropart. Phys. 04 (2015) 026.

[17] A. E. Gümrükçüoğlu, L. Heisenberg, S. Mukohyama, and N. Tanahashi, Cosmology in bimetric theory with an effective composite coupling to matter, J. Cosmol. Astropart. Phys. 04 (2015) 008.

[18] M. Lagos and J. Noller, New massive bigravity cosmologies with double matter coupling, J. Cosmol. Astropart. Phys. 01 (2016) 023.

[19] J. B. Jimenez, F. Piazza, and H. Velten, Evading the Vainshtein Mechanism with Anomalous Gravitational Wave
Speed: Constraints on Modified Gravity from Binary Pulsars, Phys. Rev. Lett. 116, 061101 (2016).

[20] G. D. Moore and A.E. Nelson, Lower bound on the propagation speed of gravity from gravitational Cherenkov radiation, J. High Energy Phys. 09 (2001) 023.

[21] R. Kimura and K. Yamamoto, Constraints on general second-order scalar-tensor models from gravitational Cherenkov radiation, J. Cosmol. Astropart. Phys. 07 (2012) 050.

[22] R. Kimura, T. Tanaka, K. Yamamoto, and Y. Yamashita, Constraint on ghost-free bigravity from gravitational Cherenkov radiation, Phys. Rev. D 94, 064059 (2016).

[23] R. N. Manchester, in On recent developments in theoretical and experimental general relativity, astrophysics and relativistic field theories. Proceedings, 12th Marcel Grossmann Meeting on General Relativity, Paris, France, 2009 (2010), Vol. 1-3, p. 226.

[24] P. Brax, A.-C. Davis, and J. Noller, Dark energy and doubly coupled bigravity, Classical Quantum Gravity 34, 095014 (2017).

[25] K. Hinterbichler and R. A. Rosen, Interacting spin-2 fields, J. High Energy Phys. 07 (2012) 047.

[26] S. Melville and J. Noller, Generalised matter couplings in massive bigravity, J. High Energy Phys. 01 (2016) 094.

[27] C. de Rham and A. J. Tolley, Vielbein to the rescue? Breaking the symmetric vielbein condition in massive gravity and multigravity, Phys. Rev. D 92, 024024 (2015).

[28] A. Matas, Cutoff for extensions of massive gravity and bi-gravity, Classical Quantum Gravity 33, 075004 (2016).

[29] L. Heisenberg, More on effective composite metrics, Phys. Rev. D 92, 023525 (2015).

[30] Q.-G. Huang, R. H. Ribeiro, Y.-H. Xing, K.-C. Zhang, and S.-Y. Zhou, On the uniqueness of the non-minimal matter coupling in massive gravity and bigravity, Phys. Lett. B 748, 356 (2015).

[31] L. Heisenberg, Non-minimal derivative couplings of the composite metric, J. Cosmol. Astropart. Phys. 11 (2015) 005 .

[32] B. F. Schutz, A First Course in General Relativity (Cambridge University Press, Cambridge, England, 2009), p. 406.

[33] T. Damour and G. Esposito-Farese, Tensor multiscalar theories of gravitation, Classical Quantum Gravity 9, 2093 (1992).

[34] K. Yagi, D. Blas, E. Barausse, and N. Yunes, Constraints on Einstein-Æther theory and Hořava gravity from binary pulsar observations, Phys. Rev. D 89, 084067 (2014).

[35] D. Bettoni, J. M. Ezquiaga, K. Hinterbichler, and M. Zumalacárregui, Gravitational waves and the fate of scalar-tensor gravity, Phys. Rev. D 95, 084029 (2017).

[36] I. Sawicki, I. D. Saltas, M. Motta, L. Amendola, and M. Kunz, Non-standard gravitational waves imply gravitational slip: On the difficulty of partially hiding new gravitational degrees of freedom, Phys. Rev. D 95, 083520 (2017). 